\title{
Die Rolle des Herausgebers der Zeitschrift Kmetijske in rokodelske novice bei der Entwicklung einer einheitlichen slowenischen Schriftsprache
}

\author{
MARKO JESENŠEK* \\ Univerza v Mariboru, Filozofska fakulteta, Koroška cesta 160, SI-2000 Maribor, Slovenija \\ Slovenska akademija znanosti in umetnosti, Novi trg 3, SI-1000 Ljubljana, Slovenija
}

Received: 2 October 2020 - Accepted: 23 November 2020

(C) 2021 The Author

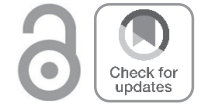

\section{ZUSAMMENFASSUNG}

Die Zeitschrift Kmetijske in rokodelske novice, herausgegeben von J. Bleiweis, spielte bei der Entwicklung der einheitlichen slowenischen Schriftsprache Mitte des 19. Jahrhunderts eine bedeutendere Rolle als bisher angenommen. Sie prägte die Kulturentwicklung im slowenischen Sprachraum langfristig; als Publikationsorgan stand sie allen Slowenisch schreibenden Autoren zur Verfügung, für Leser stellte sie eine kleine Schreibschule sowie eine Motivation zum Lesen slowenischer Texte dar.

$\mathrm{Zu}$ ihren kulturell äußerst bedeutenden Leistungen zählen die Stärkung und Fortentwicklung des Slowenentums, die Unterstützung des politischen Programms des Vereinten Sloweniens, die Ausgestaltung einer einheitlichen slowenischen Schriftsprache und Etablierung einer einheitlichen slowenischen Schrift (slovenica), die Ablehnung von Sprachideen der illyrischen bzw. panslawischen Bewegung, mit der Zeit ebenso die Festlegung von so genannten neuen Formen, abgesehen davon, dass diese vom Herausgeber Bleiweis zunächst als Widerspruch gegen „unserer reinen“ und „verständlichen“ slowenischen Sprache erklärt wurden.

Bleiweis Bemühungen um die Durchsetzung des Slowenischen in Amt und Bildung resultierten in der Ausgestaltung entsprechender funktionaler Varietäten; diese umfassten die allgemeinsprachliche Kommunikation in ,häuslicher Umgebung “, den Sprachgebrauch in der Publizistik, die fachsprachliche Ausprägung als Ergebnis der Übersetzung des Grundgesetztes (Državni zakonik) sowie die Literatursprache, dies vor allem durch die Veröffentlichung der Poesie von France Prešeren.

Zur breiten Anerkennung und Hochachtung von Bildungswesen, Zeitschriften und Büchern trug die Zeitung wesentlich bei. Ihr Herausgeber Bleiweis verstand sie zwar als „Bildungsblatt für einfaches Volk“, jedoch wurde die Zeitung auch zum politischen Periodikum für gebildete Leserschaft. Sie stand im Zentrum der

*Corresponding author. E-mail: marko.jesensek@um.si 
slowenischen Renaissance und förderte öffentliche Diskussionen über alle relevanten Fragen des Slowenentums jener Zeit, insbesondere über sprachliche, kulturpolitische und literarische Fragen.

\section{SCHLÜSSELWÖRTER}

Geschichte der slowenischen Sprache, J. Bleiweis, Kmetijske in rokodelske novice, Vereinheitlichung der slowenischen Schriftnorm, Neuslowenisch, Zedinjena Slovenija, Sprache in Amt, Sprache in Bildung

Die Rolle von J. Bleiweis, Herausgeber der Zeitschrift Kmetijske in rokodelske novice ist bei der Entwicklung einer einheitlichen slowenischen Schriftsprache bedeutender als bisher angenommen. Mit der Zeitschrift bemühte er sich um die Bildung des einfachen Volkes, denn es war ihm bewusst, dass die kulturelle Entwicklung der Slowenen nur auf einer systematischen und gründlichen Ausbildung der breiten Bevölkerungskreise gründen kann. Die Zeitschrift Novice übernahm dabei volle Verantwortung und prägte die Kulturentwicklung im slowenischen Sprachraum langfristig. Für das Bildungskonzept der Zeitschrift schien die Poesie des romantischen Dichters F. Prešeren zu anspruchsvoll und wurde deswegen eher selten veröffentlicht. Das heißt jedoch nicht, dass Bleiweis den Dichter nicht wertschätzte - trotz der Tatsache, dass er sich gegenüber der erotischen Poesie von Prešeren gelegentlich auch abwertend geäußert hatte, vgl.: „ob priložnosti sam svetohlinsko zavil oči in obsodil pesniško erotiko samega Prešerna“ (PRIJATELJ 1955: 248). Laut Historiker I. Prijatelj und Literaturhistoriker A. Žigon mochte er ihn nicht geringer als J. V. Koseski, zu jener Zeit, also Mitte des 19. Jahrhunderts, den berühmtesten slowenischen Dichter. ${ }^{1}$ Diese eher negativ wertende literaturhistorische Beobachtung ist jedoch als unbegründet zu betrachten und soll ergänzt werden. Es steht außer Frage, dass Bleiweis die slowenische kulturelle und politische Geschichte im 19. Jahrhundert bedeutend und entscheidend geprägt hat. Infolgedessen soll seine Rolle bei Ausbau der slowenischen Kulturpolitik, Stärkung des Slowenentums und Ausgestaltung einer einheitlichen slowenischen Schriftsprache gründlich beschrieben und ohne ideologische Brille analysiert werden - Bleiweis verdient heute neue, synthetische, interdisziplinäre Untersuchungen:

V prvi vrsti je nujno vsestransko raziskati praktično jezikovno dejavnost Novic, kvaliteto ubeseditve raznovrstnih vsebinskih sporočil, iz katerih je v časovnem razdobju (od ustanovitve 1.1843 pa do urednikove smrti l.1881) razvidna uspešna razvojna krivulja postopnega, namernega kultiviranja slovenskega knjižnega jezika na vseh ravninah jezikovnega (slovničnega in leksikalnega) sistema, kot tudi funkcijskega in stilnega oblikovanja tematsko motiviranih knjižnih jezikovnih zvrsti od publicistične do leposlovne, zlasti pa strokovnih (OrožEn 1996a: 154).

\footnotetext{
1 Die meist negative und unverständlich düstere Haltung gegenüber Bleiweis findet man beim Prešeren-Forscher Avgust Žigon, der den „Vater des Volkes“ lediglich nach seiner Beziehung zu Prešeren und Koseski beurteilte und andere Kriterien völlig vernachlässigte (ŽIGON 1920: 462-465). Vasilij Melik, der Historiker, bekannt nach seiner ganzheitlichen Erforschung des kulturellen Wirkens von Bleiweis, lehnte Žigons Urteil berechtigt ab (MeLIK 2002: 193); die Anbetung von Prešeren und Levstik sollte bei manchen Forschern ihr Urteilsvermögen getrübt haben (vgl.:, ,[o]boževanje Prešerna in Levstika [je] mnogim raziskovalcem zameglilo pogled“), sodass Bleiweis zur Ursache für alles Schlimme und Unerwünschte in der slowenischen Kulturpolitik wurde.
} 
Versteht man das kulturelle Programm der Zeitschrift Novice und dem Herausgeber Bleiweis seine schüchtern-heuchlerische Haltung gegenüber der erotischen Poesie, welche mit der erzieherischen Bildungsrichtung der Zeitschrift einfach nicht vereinbar war, nicht vorhält, so ist das Urteil von Žigon leicht abzulehnen. Das literarische Programm gründete auf dem Konzept des Idyllischen; Bleiweis setzte sein Vertrauen in einfache Gedichte mit nationalitätsförderndem und patriotischem Inhalt, ebenso in Anekdoten sowie humorvollen und moralistischen Geschichten. Der Hausautor der Zeitschrift war von Amts wegen J. V. Koseski, aber auch die Poesie von Prešeren wurde gelegentlich veröffentlicht. ${ }^{2}$ Allerdings sollte Prešeren für die Leser der Zeitschrift Novice inhaltlich wie auch ästhetisch einfach zu anspruchsvoll sein (OrOžEN 1996a: 166). Es handelte sich dabei um einen „besonderen sozialen Konservatismus“ der Krainer, um ihre kulturpolitische Unfindigkeit, Müßigkeit und nationalpolitische Flaute:

Gotovo je vsaj starejše generacije kranjskih Slovencev vezala s kranjskimi Nemci tradicija iste deželne pripadnosti, istih interesov kranjske dežele. Marsikaj tistega »zločestega duha«, o katerem je govoril Žigon, ne izvira od Bleiweisa, ampak je bila značilnost Kranjske, ki je dala svoj pečat seveda tudi Bleiweisu (MeLIK 2002: 196).

Bleiweis war allerdings kein Gegner der Kultivierung und des Bildungsfortschritts der slowenischen Bevölkerung. Er setzte sich für einen gebildeten Bauer und Handwerker ein, der imstande wäre, sich das nötige Wissen für seine Tätigkeit schnell anzueignen. Er hatte ein klares Ziel vor Augen: Bauer und Handwerker sollten gebildet und kultiviert werden, um sich auch des Slowenentums, also der slowenischen Sprache sowie der Bemühungen um die Ausgestaltung der slowenischen Nation im Rahmen eines selbständigen Staatsgebildes voll bewusst zu sein. Überholte traditionelle Denkweisen sollten abgelegt und die länderbedingte Zersplitterung des slowenischen Sprachraumes überwunden werden, Vorbereitungen für den nationalen Kampf sollten getroffen werden. Demzufolge gebührt Bleiweis besondere Auszeichnung für seine kulturelle und politische Wirkung zu Gunsten der slowenischen Nation im 19. Jahrhundert (MeLIK 2002: 199).

\section{BLEIWEIS ZWISCHEN KRAINISCH UND NEUSLOWENISCH}

Zu Anfang seiner publizistischen Herausgebertätigkeit (1843) wurden Bleiweis seine mangelhaften Slowenischkenntnisse vorgeworfen, eigentlich mangelhafte Kenntnisse des Krainischen, zumal es zu jener Zeit eine einheitliche slowenische Schriftsprache noch nicht gab. Angeblich

2 Mit Prešeren's Genehmigung wurde 1844 der Nachdruck seiner Dichtung Krst pri Savici veröffentlicht: „[...] zato smo slavniga gosp. pisatelja prosili, de bi nam pripustil, jo tudi v naših Novicah natisniti. Prav rad nam je dovolil, karsmo ga prosili“. Das epische Gedicht erschien in sieben Folgen auf den ersten Seiten der Zeitschrift (vom 17. Juli bis zum 28. August), samt einer Anmerkung, woraus hohe Wertschätzung für den Dichter nachvollziehbar ist: „Ne poznamo je v krajnskimu pismenstvu lepši zverstene povesti v verzih, kakor je Kerst per Savici, ki jo je naš slavni pevec, gospod Dr. Prešerin spisal in v letu 1836. dal natisniti. [...] Ta povest bo našim bravcam živ porok, de imajo kaj posebno lepiga od pesem pričakovati, ki jih bode, kakor smo slišali, gospod Dr. Preširen v kratkim na svetlo dal“ (Novice, 17.7.1844, 2/29: 114). Im Jahr 1848 veröffentlichte Bleiweis das Gedicht Zdravljica (Novice, 6.4.1848, 6/17: 69-70), und zwar auf der ersten Seite und parallel mit dem Gedicht Svobodni Lenart, verfasst vom geschickten steirischen Dichter und Geistlichen Jožef Hašnik. Darüber hinaus wurden manche Gedichte von Prešeren mit geänderter Betitelung bekannt gegeben, so z. B. Vojaška, Orglar, Memento mori, V spomin Valentina Vodnika, Od železne ceste. 
konnte er sogar in seiner Muttersprache Deutsch schriftlich nicht korrekt kommunizieren, vgl.: „Znal niti gladko pisati svoje književne materinščine“ (PRIJATELJ 1955: 244). Beim Erlernen des Krainischen verließ er sich auf die slowenische Grammatik von Metelko aus dem Jahr 1825 (METELKo 1825) ${ }^{3}$ und bediente sich dazu der sprachlichen Hilfe von Luka Jeran und Franc Malavašič (LEGIŠA 1959: 169). Der Letztere übersetzte seine anfangs auf Deutsch verfasste Texte für Novice (PrIJATELJ 1955: 244). Solange Bleiweis das Krainische noch nicht gut beherrschte, ${ }^{4}$ bei ihm zuhause wurde nämlich Deutsch gesprochen, ging er mit dem Sprachgebrauch in seiner Zeitschrift äußerst umsichtig vor und setzte sich für eine „verständliche Sprache“ ein. Das sprachliche Bild der Zeitschrift war deshalb vielfältig; angesichts der vielfältigen eingereichten dialektalen Texte wurde eine sprachliche Lösung gesucht, die für möglichst breite Leserkreise annehmbar wäre. Das Krainische erlernte Bleiweis relativ schnell (LEGIŠA 1959: 169). Durch die Zeitschrift förderte er eine überdialektale Ausprägung des Slowenischen und ermöglichte dadurch die Ausgestaltung einer einheitlichen slowenischen Schriftsprache. Durch diesen Umstand wurde das national wichtige Ziel der sprachlichen Entwicklung gesetzt (LoKAR 1909: 11) und mit Nachdruck gefordert:

Bolj pa, ko se bomo s pisanjem med seboj znanili, bolj se bomo različnih narečij navadili. Le pero v roke, dragi prijatli! In pisajte v maternem jeziku prav veliko koristnih reči za svoje rojake in hvaležni vam bodo oni in njihovi vnuki. Dokler bomo pa premišljevali, kako de bi mogli pisati, bomo zmiraj zadej ostajali. Ta je ravno žalostna na Slovenskim, de imamo tolikanj pismoukov, pa le malo pisavcov, ki bi pisali koristne reči razniga zapopadka, katerih nam tako zlo pomanjkuje (Novice, 15.1.1845, 3/3: 10).

Trotz alledem blieb Bleiweis hinsichtlich der Sprache ziemlich nachgiebig, denn seine Hauptsorge war dem Inhalt der Texte und nicht deren sprachlicher Form gewidmet. Zumal er kein Sprachforscher und nicht einmal ein „Sprachtalent“ war (BREZNIK 1933: 75), traf er selbst keiner Art sprachbezogene Entscheidungen (LoKAR 1909: 131). Als Herausgeber und Redakteur lenkte er dagegen Diskussionen, sichtete verschiedene Stellungnahmen, nahm tatsächliche sprachliche Möglichkeiten wahr und schlug daraufhin Lösungen für das (Neu)slowenische vor. Lokar äußerte Kritik an Bleiweis lediglich wegen des Synkretismus in der Benennung der „neuen“ Sprache (die krainische / slowenische Sprache), der bereits in der ersten Nummer von Novice vorkam dem Redakteur warf er scharf vor, zu jener Zeit von einem nationalen Bewusstsein zu sprechen, da dies zu früh wäre (LoKAR 1909: 129). Als würde Lokar den Geist der Zeit vor der Zeitschrift Novice nicht kennen, als würde er sozialpolitische Verhältnisse in Krain um 1848 ignorieren. Diese beschreibt Josip Vošnjak und macht dabei auf eine natürliche Zweisprachigkeit und nationale Unbestimmtheit in Krain jener Zeit aufmerksam:

Učni jezik je bil nemški, a s kmetskimi otroki, ki so govorili le slovensko, je učitelj tudi slovensko govoril. Mi iz trga smo večinoma že od doma znali nemško. V naši hiši smo med seboj navadno nemško govorili, toda znali smo tudi slovensko. Čutili pa se nismo ne za

\footnotetext{
3 Noch in den späten 70-er Jahren des 19. Jahrhunderts verlangte er eine einfache und verständliche Sprache und die Orientierung an der Grammatik von Metelko. Sprachliche Neuigkeiten lehnte er entscheidend ab, vgl.: „tistih novotarij“, ki jih uporabljajo ,taki, ki sicer malo delajo, pa se radi guncajo po specijalitetah. Meni je Metelkova slovnica sveta slovnica, katere še nikdo ni prekosil“" (Novice, 1883, 41/24: 192).

4 Das geschriebene Krainisch vervollständigte er ebenso im Priesterseminar (PRIJATELJ 1955: 244). Junge Theologen belehrten ihn der funktionalen Vielfältigkeit der Sprache und begeisterten ihn für das Krainische.
} 
Nemce, ne za Slovence, ker se za narodnost sploh nikdo ni menil do 1.1848 in nam je jezik bil le sredstvo, da se sporazumemo med seboj in z drugimi (VOŠNJAK 1982: 15).

Deutlich positiver äußerte sich über Bleiweis der slowenische Literaturhistoriker Josip Marn; er nannte ihn zum Nachfolger von Valentin Vodnik (MARN 1882: 1) und hob hervor, dass in der Zeitschrift Novice bereits im Jahr 1844 über Slowenen als Vertreter einer einheitlichen Nation gesprochen und eine gemeinsame slowenische Schriftsprache befürwortet wurde. Dies ist an der Stellungnahme von Bleiweis nachvollziehbar, seine Zeitschrift sei nicht nur für Krainer in Oberund Unterkrain gedacht, sondern sie sei „allen Slowenen“ gewidmet. 1845 begrüßte Bleiweis das „steirische Slowenisch“ und Bemühungen von A. Krempl um eine einheitliche slowenische Sprache. Durch die traditionsreiche religiöse Literatur in Slowenisch sprechenden Gebieten war dieses Ziel relativ leicht zu erreichen, während im profanen historischen Werk von Krempl mit dem Titel Dogodivšine Štajerske zemle (1845) weiterhin eine doppelte morphologisch-syntaktische und lexikale Ausprägung des Slowenischen nachvollziehbar ist. Darüber hinaus warnte Bleiweis vor wortwörtlicher Übersetzung aus dem Deutschen; „der Geist der slowenischen Sprache“ sollte bei der Übersetzung verfolgt werden, deutsche Inhalte sollten im slowenischen Gewand präsentiert werden (MARN 1882: 15), ganz nach dem alten Sprichwort, auf welches sich Bleiweis noch im Jahr 1855 berufen hat: „Čerka le mori, duh pa živi!“ (Novice, 5.5.1855, 13/36: 144). Sprachliche Neuigkeiten sollte man mit Geduld wahrnehmen und nur dann akzeptieren, wenn sie auch unter den Sprechern breite Verwendung und Bestätigung finden, wenn sie so gut wie alter Wein werden: „Naj vre mošt, da se izdela stanovitno vino!“ (Novice, 5.5.1855, 13/36: 143). Marn schätzte die vorsichtigen und besonnenen sprachlichen Ansichten von Bleiweis (Festina lente! / Hiti počasi!), weil sie sich auch bei der Durchsetzung der heutigen slowenischen Schrift (Slovenica) bestätigt haben. Die Vereinheitlichung der slowenischen Sprache samt Schrift führten Bleiwes zur Erkenntnis, dass die Grammatiken von F. S. Metelko, B. Potočnik, M. Murko und A. Janežič den neuen Zuständen nicht mehr gerecht werden können (METELKo 1825, Ротос̌NiK 1849, Murko 1832, JANEžIČ 1854). Infolgedessen kam er 1859 mit der Idee, eine neue Grammatik zu verfassen, um unendlichen sprachbezogenen Streitigkeiten ein Ende zu bereiten. Diese Erkenntnis war die Folge seiner Auseinandersetzungen mit verschiedenen Sprachpositionen der Fachleute und vieler Sprecher des Slowenischen sowie seiner „Konsultationen“ bei Matija Čop (welche von Žigon verschwiegen, obgleich sie bereits bei Marn erwähnt wurden, vgl. MARN 1882: 57), der ihn belehrte, dass das Nachdenken über die Sprache kein Ende kennt und von großer Wichtigkeit ist. Radoslav Razlag, slowenischer Dichter, Schriftsteller und Politiker lehnte solche Positionen von Bleiweis ab, vgl.: „Dieser Mann versteht keine Sache gründlich!“; noch entschiedener äußerte sich dazu Žigon. Dessen scharf negatives Urteil über Bleiweis milderten erst die Grammatiker Anton Breznik und daraufhin Jože Toporišič. Obgleich Breznik fest überzeugt war, dass Bleiweis keine fundierten Sprachkenntnisse besaß und keine Belesenheit und noch weniger Ausbildung hatte (Toporišič 1986: 93), respektierte er die Veröffentlichung einiger bedeutender fachlicher Abhandlungen über das Slowenische in der Zeitschrift Novice, darunter den Aufsatz Napake slovenskega pisanja von Fran Levstik (Levstik 1858), der von Breznik wohlwollend beurteilt und als ,ein positives Schreiben gegen die Germanisierung “ interpretiert wurde (BREZNIK 1982: 281). Laut Bleiweis trugen Novice zur Ausbesserung des Slowenischen bei, während andere damalige slowenische Zeitschriften sowie nichtpublizistische Meinungen zur Sprache von ihm abgelehnt wurden. Toporišič hielt diese abneigenden Stellungnahmen zu Pressekonkurrenz und Andersdenkenden bei Bleiweis als Absolutismus (BREZNIK 1982: 92), den er allerdings befürwor- 
tete, zumal sich dadurch das regional-zentrale Krainische als Grundlage für das Neuslowenische durchsetzen konnte, vgl.: „Kranjski bi bil pravzaprav ta jezik lahko tudi ostal, ko bi bil umel pritegniti večino nekranjsko pišočih, ne le posameznikov, kakršnih eden je bil pri Štajercih Anton Murko, ki ga poznamo že kot nasprotnika dajnčice in metelčice“ (BREZNIK 1982: 277).

\section{VEREINIGTES SLOWENIEN (ZEDINJENA SLOVENIJA) UND DIE VEREINHEITLICHUNG DER SLOWENISCHEN SCHRIFTSPRACHE}

Die Zeitschrift Novice sprach seinerzeit die gesamte slowenische Bevölkerung an, heute gilt sie als historische Quelle der slowenischen nationalen Wiedergeburt und Staatsbildung Mitte des 19. Jahrhunderts. Bleiweis als Herausgeber und Redakteur stand hinter Allem, was die Zeitschrift darstellte: „[... Že za tega delj, da mu je poklonil 'Novice', zasluži po vsej pravici, da ga prištevamo pervim dobrotnikom domovine slovenske" (Levec 1880: 10). Unter seiner Redaktion wurde sie zu einem universellen slowenischen Blatt (TOMINŠEK 1904: 654) - in bescheidenen Umständen entstanden und mit der Zeit zu einer national-kulturellen Tribüne des jungen Volkes geworden (Prijatelj 1955: 244), damit sich dieses die slowenische Sprache, Literatur und Kultur als die bedeutendsten physischen und geistigen Elemente des Slowenentums bewusst machen konnte. Bleiweis unterstrich die Notwendigkeit einer einheitlichen slowenischen Sprache, hob ihre Eigenständigkeit hervor und erkannte darin das einzig taugliche und natürliche Mittel zur gegenseitigen Kommunikation sowie zur Vereinigung und Ausbildung aller Slowenen (OrožEN 1996a: 153). Entschlossen, obgleich die Entschlossenheit nicht zu seinen Charakterzügen zählte, setzte er sich für die Kultur, Ausbildung und Erziehung in der Muttersprache ein - in diesen Bereichen sah er einen natürlichen Vorrang der Muttersprache vor dem Deutschen; hätte die Fremdsprache einen unbegrenzten Primat vor der Muttersprache, so wäre es, wie „s cerkvijo okoli križa hoditi, a ne s križem okoli cerkve“ (MARN 1882: 57). Bleiweis' Verdienste für die durch eine einheitliche Sprache bedingte Verbundenheit der Slowenen, welche mittels Novice mitgestaltet wurde, können nicht als „patriotische 'schöne Lüge'“ (PRIJATELJ 1955: 170) bezeichnet werden; es ging ihm um die öffentliche Bestätigung der Eigenartigkeit der slowenischen Sprache und an diesem ehrlichen Kampf lassen sich auch seine eigenen Gedanken und Emotionen nachvollziehen. Sein Lebensweg ist mit der Fortentwicklung des Slowenentums und mit dem Programm des Vereinten Sloweniens auf engste verbunden. Allerdings wurden die Slowenen aus Prekmurje aus dem Programm ausgeschlossen, denn es ist anzunehmen, dass Bleiweis Mitte des 19. Jahrhunderts über sie nicht viel oder gar nichts wusste (LONČAR 1909: 169). Die Ausgestaltung einer einheitlichen slowenischen Sprache wollte er in engem Verhältnis mit der politischen Idee des Vereinigtes Sloweniens sehen; allmählich akzeptierte er diese vorbehaltlos und bezog programmmäßig auch das Neuslowenische mit ein. Am 6. April 1848 präsentierte er sein sprachpolitisches Programm in der krainischen Landesversammlung als Anspruch der Slowenen 1) auf die slowenische Lehrsprache in der Grundschule auf dem Lande; 2) auf stärkere Berücksichtigung des Slowenischen an den städtischen Schulen; 3) auf die Einführung des Slowenischen als Lehrfach an Gymnasien; 4) auf die Einführung der Schrift Gajica; 5) auf das Amtieren in slowenischer Sprache. Hiermit zeigten sich die ersten Abrisse der slowenischen Nation sowie Bemühungen um die Benutzung des Slowenischen in Bildung und Amt. Prijatelj sah im Auftritt von Bleiweis nur ein erstes vorsichtiges Manöver, „ut aliquid fecisse videatur“; er handelte berechnend, indem er seine nationalen Ideen 
dem Großherzog Johann als jene des Illyrischen Königtums vorstellte (PRIJATELJ 1955: 61). Prekmurje und seine Ausprägung der slowenischen Sprache waren nicht mitgedacht:

Nekateri za povzdigo naše narodnosti vneti Slovenci žele, de bi se kranjska dežela, slovenski del koroške in štajerske dežele, slovensko Istrijansko in Goriško v en narod zedinili. Mi smo te želje nekimu presvitlimu Gospodu na Dunaji razodeli, ki so jih pravične poterdili in pohvalili [...]. Kranjci bojo - po mojih mislih - slovenskim bratam Štajerskiga, Koroškiga, Istrijanskega, Goriškega s serčnim veseljem roko podali in se z njimi zedinili, de bi bila Ljubljana sredica slovenskih dežela [...] (Novice, 19.4.1848, 4/16: 66).

Die Zeitschrift Novice teilte mit, dass Ideen des Illyrismus nicht verwirklicht werden, während der Gedanke über das vereinigte Slowenien erst im Jahr 1860 wieder angesprochen wurde. Diesmal handelte Bleiweis viel entschlossener. Er sammelte Unterschriften für die Petition über die slowenische Sprache, in der Überzeugung, das Recht zum Gebrauch der Muttersprache zählt zu den Grundrechten eines jeden Menschen. Sprachlichen Ideen aus dem Jahr 1848 fügte er Anforderungen an eine administrative Vereinigung der slowenischen Länder hinzu: 1) Grundschulen funktionieren auf nationaler Basis, mittlere Schulen beachten die Gleichsetzung der slowenischen, deutschen und italienischen Sprache; 2) Vertreter der Obrigkeit berücksichtigen Sprachpräferenzen des Einzelnen; 3) gerichtliche Bekanntmachungen erscheinen ausnahmslos in slowenischer Presse; 4) slowenische Länder werden zu einer administrativen Einheit (Novice, 1.5.1861, 19/18: 144-145). Die slowenische National- und Sprachfrage wurden somit gleichgesetzt. In Bildung und Amt wurde die volle Inkraftsetzung des Slowenischen verlangt, die Muttersprache wurde zum „natürlichen und kürzesten Weg“ zur Bildung, Erziehung und Kultur erklärt, „ker je materinščina najterdnejša podlaga za vsaki drugi jezik in nauk, in naposled, ker je politična in sodna uprava najcenejša, najpravičnejša in najložja, ako se opravlja v jeziku narodovem [...]" (Novice, 6.11.1861, 19/45: 367). Bei einer administrativen Vereinigung aller slowenischen Länder wäre die sprachliche Gleichsetzung in Bildung und Amt leichter zu erreichen. D. Kermauner interpretierte die Verengung der Nationalfrage lediglich auf die Verteidigung der Sprache als „hilflose Beschränktheit", zumal dadurch eine staatsbildende Position gegenüber Wien und seinem Kaiser beeinträchtigt werde (Kermauner 1956: 545). Die Ideen von Bleiweis wurden allerdings nicht allgemein akzeptiert; unter anderem waren die sog. nemškutarji (Deutschtümler) dagegen:

[...] visoko inteligentni in aktivni možje, ki so se v mladih letih samostojno izobrazili v materinščini in vzljubili njeno kulturo, ki pa so v teh letih obnavljajočega se ustavnega življenja odpadli od slovenske stvari, se pridružili nemški stvari in postali voditelji ne samo prvih dveh skupin svojih nemškutarskih rojakov, ampak tudi tretje, po poreklu tuje kategorije kot idejni inspiratorji tiste smeri in faktorja slovenskega kulturnopolitičnega življenja, ki ju poznamo v naši preteklosti pod imenom »nemškutarstvo« (PRIJATELJ 1956: 61).

Bleiweis verstand das Vereinigte Slowenien so, „da domači niso tuji v lastni domovini“, und diese Position setzte die Etablierung des Slowenischen in Bildung und Amt sowie im öffentlichen Leben voraus; im Rahmen des einheitlichen Österreichs strebte er lediglich Landesautonomie, die Beachtung des historischen Rechts und der nationalen Rechte an. Zu einem selbstständigen Staatsgebilde konnte ein derartiger Austroslavismus allerdings nicht führen. Die größte Errungenschaft des idealen vereinigten Sloweniens (PrIJATELJ 1956: 100) war die Wiener Anerkennung 
der Slowenischen Sprache ${ }^{5}$ - am 4. März 1849 wurde durch einen Erlass festgelegt, dass das „österreichische Grundgesetz" aus dem Deutschen ins Slowenische übersetzt werden soll. Folglich wurde die Übersetzung des Grundgesetzes (Državni zakonik) zu „Rechtschreibung, Wörterbuch und Grammatik“ der einheitlichen slowenischen Schriftsprache. Im November 1849 erschien die erste Nummer von Zakonik. Der Übersetzer Franc Miklošič führte die sog. „neuen Formen“ ein, ${ }^{6}$ die als äußeres erkennbares Merkmal des Neuslowenischen galten (OROŽEN 1996b). Bleiweis selbst war darüber nicht begeistert (PRIJATELJ 1955: 77) und benutzte sie erst nach Jahren, in der Zeitschrift Novice setzten sie sich jedoch allmählich durch.

\section{DIE SLOWENISCHE AMTSSPRACHE}

In Krain überwogen schließlich Deutschtümler, für welche die slowenische Amtssprache und slowenisch sprechende Beamte unnötig waren. Infolgedessen wurden Slowenischkurse für Beamte und Angestellte immer seltener und unter dem Absolutismus Bachs verlor die Verwendung der slowenischen Amtssprache an der Bedeutung (PRIJATELj 1955: 77). In den 60-er Jahren des 19. Jahrhunderts schien es, dass der Kampf um die Slowenisch sprechende Beamtenschaft völlig gescheitert wäre.

Gemäß dem Erlass des Ministers Belcredi vom 30. Juli 1865 sollte in mehrsprachigen Gebieten Österreichs das Amtieren in der jeweiligen Landessprache gesichert sein. Bleiweis zeigte Vertrauen in diese Verordnung, allerdings blieb er weiterhin sehr vorsichtig und hoffte auf ihre tatsächliche Durchsetzung. In der Zeitschrift Novice wurden Texte in der slowenischen Amtssprache vorübergehend nicht veröffentlicht. In der Fortsetzung der Bemühungen um die Etablierung des Slowenischen als einheitliche Landessprache stand die Einführung des Slowenischen in verschiedene Bildungsinstitutionen im Vordergrund, um Slowenisch sprechende Beamte ausbilden und die slowenische Bevölkerung an sich kultivieren zu können. Bleiweis wie auch F. Levstik waren sich einig, dass eine allseitige Kultur des Volkes mehr als nur „einfache Büchlein“ verlangt, wofür jedoch bei der dialektalen Vielfältigkeit des slowenischen Sprachgebietes eine breit akzeptierte

\footnotetext{
5 F. Miklošič gebührt das Verdienst, die amtlich-staatliche Anerkennung einer einheitlichen Benennung des Slowenischen mit dem Ausdruck slovenski, anfangs auch novoslovenski, also slowenisch bzw. neuslowenisch zu erlangen. Dadurch wurde eine einheitliche Schriftnorm der slowenischen Sprache festgelegt und zugleich der amtlichrechtliche Status für die Sprache der Slowenen ohne nationale Anerkennung in der Monarchie gefestigt. In der Zeitschrift Slovenija fand sich eine knappe anonyme Notiz darüber: „Grenka je bila kupica, pa spili smo jo - die slowenische Sprache ist amtlich anerkannt".

6 Neue Formen wurden ab 1852 gänzlich akzeptiert; bis Ende des 19. Jahrhunderts setzten sich folgende durch: Substantivische Suffixe: - $\boldsymbol{a m},-\boldsymbol{a m a} \rightarrow-\boldsymbol{o m},-\boldsymbol{o m a}$ (bratam, bratama $\rightarrow$ bratom, bratoma); Umlaute nach $c, z, \check{c}, \check{s}, \check{z}$, j: -om, -oma $\rightarrow$-em, -ema (stricom, stricoma $\rightarrow$ stricem, stricema); adjektivische Suffixe: -iga, -imu, -im $\rightarrow$-ega, -етu, -ет; (lepiga, lepimu, lepim $\rightarrow$ lepega, lepemu, lepem); Genusdifferenzierung beim Komparativ: $-\boldsymbol{i} \rightarrow-\boldsymbol{i},-\boldsymbol{a},-\boldsymbol{e}$ (večji človek, večji hiša, večji mesto $\rightarrow$ večji človek, večja hiša, večje mesto); Übereinstimmung des Attributs mit dem Bezugswort in Genus, Kasus und Numerus: $-\boldsymbol{e} \rightarrow-\boldsymbol{a}$ (junaške dela $\rightarrow$ junaška dela); Interfix bei Verben: -ni- $\rightarrow$

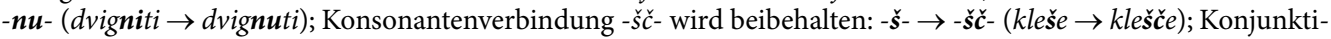
on: $\boldsymbol{d} \boldsymbol{e} \rightarrow \boldsymbol{d a}$; Präfix beim Superlativ: nar- $\rightarrow$ naj- (narlepši $\rightarrow$ najlepši); Schreibung des Halblautes: $\mathrm{z} \boldsymbol{i}$-jem $\rightarrow \mathrm{z} \boldsymbol{e}$ jem (sivic, sivčik, sim $\rightarrow$ sivec, sivče $k$, sem); Schreibung des silbenbildenden Sonanten $r:-\boldsymbol{e r} \rightarrow-\boldsymbol{r}(k \boldsymbol{e r t}$, vert, pert $\rightarrow$ krt, vrt,prt; toda: dober, oster); verbales Morphem -i (Reflex des unbetonnten Jats) $\rightarrow-\boldsymbol{e}$ (vediti, viditi, vidil $\rightarrow$ vedeti, videti, videl); adjektivisches Pronomen ves: vsiga, vsimu $\rightarrow$ vsega, vsemu; Diminutivsuffix: $-\boldsymbol{i c a} \rightarrow-\boldsymbol{c a}$ (stvarica, živalica, lučica $\rightarrow$ stvarca, živalca, lučca); Suffix -vec / -lec: -vec $\rightarrow$-lec (bravec $\rightarrow$ bralec); Einzelheiten: (grajščak, grajščina $\rightarrow$ graščak, graščina; Juri, Jurja $\rightarrow$ Jurij, Jurija; pri komur, čemur, s komur, sčimur $\rightarrow$ pri komer, čemer...).
} 
einheitliche Schriftsprache Bedingung war. F. Levstik nahm Anstoß an der Verkündigung in Novice, dass der von Franc Kosar verfasste Lebenslauf des Bischofs Slomšek auf Deutsch erscheinen sollte, um seine sprachfördernde Tätigkeit vor den Deutschen zu rechtfertigen (Naprej, 21.4.1863, 127). Beide waren der Meinung, dass die Slowenen den Fürstbischof von Lavant in ihrer Muttersprache kennen lernen sollten und dass für den kulturellen Wachstum des Volkes die Ausbildung auf Slowenisch unerlässlich sei (Naprej, 24.4.1863, 132).

Die Idee des vereinigten Sloweniens als administrative und möglicherweise auch staatsrechtliche Einheit im Rahmen des österreichischen Staates wurde am 30.8.1870 in der Krainischen Landesversammlung zum ersten Mal diskutiert. Auf Bleiweis' Anregung erfolgte im nächsten Jahr (am 14.10.1871) eine entsprechende Botschaft an die Wiener Regierung. Folgendes wurde gefordert: 1) Vereinigung aller Slowenen in einem Kronland; 2) Erweiterung der Rechte der Landesversammlung; 3) Einführung und Durchsetzung des Slowenischen in Bildung und Amt; 4) erhöhte Zahl der mittleren Schulen mit Slowenisch als Unterrichtssprache; 5) Gründung einer juridischen Fakultät in Ljubljana und Einrichtung nationaler Kurien; 6) Gründung eines höheren Landesgerichts für slowenische Regionen in Ljubljana (MAL 1993: 1009). In Folge darauf hat sich leider nicht viel geändert; die einzige, allerdings bedeutende Errungenschaft des Vereinigten Sloweniens blieben die slowenische Übersetzung des Grundgesetzes (Državni zakonik) und die Herausgabe etlicher Lehrbücher für slowenische Schulen.

\section{DIE SLOWENISCHE UNTERRICHTSSPRACHE}

Nach der Märzrevolution 1848 dauerte es mehr als zehn Jahre, bis man die slowenische Unterrichtssprache in größerem Umfang einführen und durchsetzen konnte. Bis in die 60-er Jahre wurde Slowenisch von den Vertretern und Sympathisanten des Deutschtums abgelehnt, da angeblich funktional zu wenig ausdifferenziert und den Standards einer anerkannten Schriftsprache nicht entsprechend. Die dialektale Vielfalt behinderte den öffentlichen Gebrauch, zu wenig eigener Literatur und Lehrbücher wirkten angeblich beeinträchtigend (PRIJATELJ 1956: 95). ${ }^{7}$ Bleiweis lehnte solche Unwahrheiten kategorisch ab: Mit der Aufzählung der bedeutendsten Werke der slowenischen religiösen und profanen Literatur (Trubar, Vodnik, Prešeren, Koseski, Toman) klärte er die Leser über die wahren Sachverhalte auf, er machte auf rege Aktivitäten des Verlags Mohorjeva sowie auf viele slowenische Übersetzungen der Weltliteratur aufmerksam, nannte etliche Wörterbücher und Grammatiken in lateinischer, italienischer, deutscher und slowenischer Sprache und ebenso mehrere Bibelübersetzungen. Nicht zuletzt wies er auf die berühmtesten Sprachforscher des 19. Jahrhunderts J. Kopitar und F. Miklošič hin. Dadurch zeigte er überzeugend, dass die Verfassung von schlichten Schulbüchern in einer Sprache mit umfangreicher und vielfältiger literarischer Tradition keine Schwierigkeit darstellen sollte (Novice, 9.10.1861, 19/41: 342). Die Verhältnisse kannte er eingehend und handelte auch hinsichtlich der slowenischen Unterrichtssprache nach seinen bewehrten Regeln: langfristiges Planen und kontrollierte Zulassung von Zweifeln und Bedenken. Er befürwortete den schulischen Unterricht auf Slowenisch und

7 In Novice veröffentlichte Bleiweis einen Auszug aus dem spöttischen Bericht der Wiener Zeitschrtift Presse über das „unkultivierte Slowenisch“, allerdings samt eigener entschlossener Ablehnung solcher Positionen: „[...] aber die Regierung ist nicht Schuld, wenn die an das Lallen der Kindheit mahnende Mundart Tomaniens sich noch nicht zur Bedeutung einer Cultur-Sprache erhoben hat, und wenn der Vocabeln-Reichthum dieses primitiven Dialectes kaum noch ausreicht zur Uebersetzung des kleinen Katechismus“ (Novice, 9.10.1861, 19/41: 341). 
stimmte zugleich der deutschen Sprache zu, wenn diese von den meisten Teilnehmern erwünscht sei. Vom Gymnasialunterricht erhoffte er eine gleichrangige Beherrschung des Slowenischen und Deutschen in Rede und Schrift. Um die Qualität der Slowenischkenntnisse bei Gymnasiasten zu sichern, stellte er slowenische Lesebücher für untere Gymnasialklassen zusammen. Die Herausgabe des slowenischen Lesebuchs für die erste Gymnasialklasse im Jahr 1851 (Slovensko berilo za I. gimnazijalni razred) hielt er für einen historisch äußerst wichtigen Moment, vergleichbar mit der slowenischen Übersetzung des Grundgesetzes (Državni zakonik). Im Brief des Ministers Thun vom 6.2.1851 wurde die im Lesebuch niedergeschriebene slowenische Sprache zur gesetzlich verordneten Unterrichtssprache an allen slowenischen Schulen in Österreich und somit mit der Sprache des Državni zakonik gleichgesetzt:

Ker je pa »občni deržavni zakonik in vladni list« pot nastopil, po kateri se zamorejo vse slovenske podnarečja $\mathrm{v}$ jedni književni jezik zediniti, in ker se sme pričakovati, da bo jezik postave (zakona), pospešijoč izobrazenje javniga vradniga jezika, tudi v slovstvu pervenstvo zadobil, se ne branim izreči, de ima ta jezik tui v šole vpeljan biti (Novice, 26.2.1851, 9/9: 45).

Durch die Bestätigung der slowenischen Lesebücher wurde die einheitliche slowenische Sprache gesetzlich geregelt und für den öffentlichen Sprachgebrauch auf dem gesamten slowenischen Gebiet bestimmt, während Dialekte auf die häusliche Kommunikation eingeschränkt wurden. Mit Bemühungen um die slowenische Sprache, um slowenische Schulen und slowenische Lehrbücher ${ }^{8}$ schwächte er die Befürworter des Deutschtums und Gegner einer einheitlichen slowenischen Sprache im Lande ab. Daraufhin wurden Slowenisch als Unterrichtssprache an den Volksschulen in ländlichen Gebieten sowie Slowenisch als Schulfach an den mittleren Schulen eingeführt. Sprachpolitische Bestrebungen von Bleiweis brachten allmählich positive Ergebnisse. Bis 1865 wurden 35 neue slowenische Volksschulen eingerichtet, somit waren es insgesamt 163, jedoch betrieb man in Krain weiterhin 53 zweisprachige und 17 deutsche Volksschulen (Novice, 16.8.1865, 23/33: 264). Daraufhin schlug er 1866 der Landesversammlung eine neue schulische Sprachregelung vor: Die obligatorische Unterrichtssprache an allen unteren Volksschulen, Hauptschulen und Normalschulen ist Slowenisch (ausgenommen die Gottscheer Region), in der 3. und 4. Hauptschulklasse kommt dazu Deutsch als Schulfach, in den mittleren Schulen werden sukzessive einzelne Fächer mit slowenischer Unterrichtssprache eingeführt, in Realschulen und Gymnasien erfolgt der Unterricht gänzlich auf Slowenisch (Novice, 17.1.1866, 24/3: 23). Bis Ende der 60-er Jahre des 19. Jahrhunderts konnten alle Vorschläge jedoch nicht verwirklicht werden. Die realen Zustände waren zum Teil anders: Slowenisch als Schulfach wurde auf Slowenisch unterrichtet, andere Schulfächer dagegen auf Deutsch, da die Lehrer der slowenischen Sprache sehr oft nicht mächtig waren. Wegen sprachlicher Bestrebungen war Bleiweis bei den Deutschtümlern äußerst unbeliebt und wurde sogar polizeilich angeklagt, und zwar wegen Lehrer, die angeblich

\footnotetext{
8 „Kje na celem svetu se pišejo šolske knjige, preden se ve, da bodo rabljene v šoli! Pa kdaj se je nam reklo, da naj jih pripravimo, kdaj se nam je le reklo, da jih bo treba? Ali vendar hočete zdaj od nas knjig imeti, in ker jih nimamo, ne puščate nam jezika v šolo. Knjig nam pripraviti ne daste, in brez njih nam ne vzamete jezika v šolo; tako utegnemo, verte se vedno v circulo vitioso, čakati ravnopravnosti narodne zastonj do sodnega dne. Recite samo, da je knjig treba, in katerih, ter dajte nam samo nekaj od tistih denarjev, ki jih izdajemoza nemške knjige vaše zaloge, in verjemite, čudili se bote, kako hitro si bodo Slovenci potrebne učne knjige priskrbeli“ (Novice, 9.10.1861, 19/41: 342).
} 
nicht mehr „richtig denken könnten“, da sie sich zu viel mit dem Slowenischen und zu wenig mit dem Deutschen beschäftigen müssten (MAL 1993: 915).

Nach dem Frühling der Nationen wurde auch die Idee zur Gründung einer slowenischen Universität in Ljubljana geboren. Anton Mažgon, ${ }^{9}$ der erste Übersetzer des österreichischen Grundgesetzes ins Slowenische, hielt ab dem 20. März 1849 Vorlesungen zum allgemeinen bürgerlichen Recht an der neugegründeten Rechtswissenschaftlichen Akademie in Ljubljana in slowenischer Sprache, ab Juni 1849 erfolgten ebenso slowenische Vorlesungen zum bürgerlichen Recht von Ernest Lehman. In seiner charakteristischen Art sprach sich Bleiweis für die Idee zunächst eher zögernd aus (PRIJATELJ 1956: 87-88), er wurde allerdings zum öffentlichen Befürworter der Universität, sobald sich dazu auch der Bildungsminister Franc Serafin Stadion positiv geäußert hatte. Nach dessen Abtritt 1849 wurde der Vorschlag zurückgezogen, während beide slowenisch vorgetragene Fächer in das Studienprogramm der Grazer Universität integriert wurden.

Die ersten slowenischen Hochschulvorlesungen deutete Bleiweis als "glückliches Zeichen“ und verstand sie als Ankündigung einer „allmählichen“ Gründung der Universität in Ljubljana. Bis dahin setzte er sich für die Vorlesungen auf Slowenisch zumindest im Bereich des Zivil- und Strafrechts ein, damit eine leichtere Verständigung der Rechtsbeamte mit dem einheimischen Volk in slowenischen Ländern gesichert wäre (Novice, 26.9.1849, 7/39: 171-172). Trotz der Tatsache, dass zu jener Zeit bereits mehrere Universitätslehrer in Österreich slowenisch vorgetragen hatten (slowenische Rechtsvorlesungen hielten Josip Kranj in Graz sowie Janez Kopač und Josip Mihael Škedl in Innsbruck, an der Theologischen Fakultät in Graz lasen Matija Robič und Jožef Tosi in slowenischer Sprache vor), war die Zeit für slowenische Rechtsvorlesungen in Ljubljana noch nicht reif. Bleiweis handelte hierzu realistisch und bediente sich der bewährten Methode kleiner Schritte - zunächst war das Amtieren in slowenischer Sprache sicherzustellen und deutschtümlerische Feindlichkeit gegenüber allem Slowenischen zu übersteigen. Es war ihm bewusst, dass die österreichische Regierung nach dem Abtritt Stadions der slowenischen Universität nicht zugeneigt war, und so wandte er sich anderen leichter erreichbaren sprachlich-kulturellen und bildungsbezogenen Zielen zu, während die Gründung der Universität auf spätere Zeit verschoben wurde. Die Zeitschrift Novice berichtete hierzu, wie es scheint, ohne größeres Bedauern, zumal der neue Bildungsminister Leopold Thun-Hohenstein offensichtlich nur Deutsch als Unterrichtssprache an den österreichischen Universitäten befürwortete und mit dem Minister Bach sich die neuabsolutistische Periode ankündigte:

V saboto je bilo v nemškim Ljubljanskim časopisu oznanjeno, de imata po ministerskim ukazu v Ljubljani slovenski šoli kriminalniga in deželniga prava nehati, v kteri sta rajnki gosp. Mažgon in pa gosp. Dr. Lehman pretečeno leto učil, in da se imate te dve šoli Graškimu vseučilišu pridjati. [...] če visoko ministerstvo ni tega ukaza le začasno dalo, dokler se prihodnja vseučiliška naprava za različne kronovine za gotovo ne sklene in ne dožene: ali bo prišlo v Ljubljano vseučeliše ali ne (Novice, 26.9.1849, 7/39: 171).

9 A. Mažgon starb am 24. August 1849 an Lungenentzündung im Alter von 37 Jahren. In der Todesanzeige der Zeitschrift Slovenija wurde er als „unermüdlicher Patriot“ verabschiedet, France Cegnar widmete ihm einen Achtstrophenlied mit dem Motto: Slovenije sreča mu bilo je geslo (Slovenija, 28.8.1849, 2/69: 276). 


\section{BLEIWEIS UND DIE AUSGESTALTUNG EINER EINHEITLICHEN SLOWENISCHEN SCHRIFTSPRACHE}

Der Zeitschrift Novice kam eine wichtige Rolle bei der Ausgestaltung, Festigung und Entwicklung der einheitlichen slowenischen Schriftsprache zu. Die Besonnenheit und Geduld des Redakteurs sowie sein Wohlwollen (ŠTREKELJ 2014: 910) führten zur Verwirklichung des Gedankens von der Notwendigkeit einer gemeinsamen „nationalen“ Sprache und ihrer Durchsetzung in Amt und Bildung. Die Annahme neuer Formen und die Einführung der Schrift Slovenica erfolgten in engem Zusammenhang mit der Zeitschrift Novice - es stellt sich sogar die Frage, ob zur Vereinheitlichung der slowenischen Schriftnorm überhaupt und schon gar nicht bereits im 19. Jahrhundert kommen würde. Wenn Navratil als Pionier und Vater einer manierlichen slowenischen Konversation angesehen wird („pravi začetnik in oče olikane slovenske konverzacije“), er illustrierte nämlich, wie man miteinander slowenisch reden sollte („kako se morajo pomenkovati po slovenski naši gospodje“) und verwendete dazu selbst eine reine und flüssige slowenische Schriftsprache („[g]ovoril čisto pismeno slovenščino, ki mu je tekla gladko in sladko“) (TRDINA 1905: 721), so soll man Bleiweis bedeutende Verdienste für ihre Anerkennung und Verbreitung zuschreiben, zumal seine Zeitschrift sich als nützlich dem Volke, der Literatur und der Sprache erwiesen hatte („koristile toliko našemu narodu, slovstvu in jeziku“) (Trdina 1905: 590). Die einflussreichsten Krainer jener Zeit sprachen untereinander immer deutsch („pogovarjali med sabo vedno po nemški“), selten gehörte Dialekte waren überfüllt von Germanismen und Lokalismen (Trdina 1905: 590). Bleiweis war dabei keine Ausnahme, nur er war sich der Sprachfrage bewusst und nutzte die Zeitschrift Novice und seine Redakteurposition aus, um die slowenische Schriftsprache zu einer überregionalen und geordneten Schriftnorm zu erheben. Darüber hinaus setzte er sich für die Sprache mehrere Male auch in der Landesversammlung ein, u. a. am 6. April 1861 mit einer überraschenden Anforderung eines zweisprachigen offiziellen Schreibens an den Kaiser in Wien:

$\mathrm{V}$ dvorani se je zaradi novosti take zahteve pojavilo sicer hipno vznemirjenje, vendar pa se nikdo ni upal ugovarjati Bleiweisovemu utemeljevanju, da mora za vsebino zvedeti tudi prebivalstvo dežele, ki je ki je po dveh tretjinah slovensko. V tej adresi na cesarja je deželni zbor poudaril, da more moč in varnost države zagotoviti le tako edinstvo, ki dopušča notranjemu domačemu življenju posameznih dežel glede na njihove narodnostne posebnosti v šegah, omiki in pravni zavesti svoboden razvoj (MAL 1993: 1038).

Die Ansichten von Bleiweis zum Sprachgebrauch in der Bildung und Öffentlichkeit waren seinerzeit sehr fortschrittlich und könnten stellenweise noch heute vorbildhaft sein. So berief er sich 1868 auf den Artikel 19 des Grundgesetzes, wonach eine jede Nation in Österreich das Recht zur Gesetzgebung in nationaler Sprache besitzt. Dementsprechend verlangte er eine sprachliche Gleichberechtigung des Deutschen und Slowenischen in krainischen Schulen und Ämtern, wonach das Slowenische in slowenischsprachigen Gebieten den Status der Amtssprache erhielt. Die damals bestehende amtliche und schulische Zweisprachigkeit wäre somit aufgehoben. Derartige Ansichten lassen sich teilweise auf gegenwärtige sprachpolitische Zustände in Slowenien übertragen; besonders in der (höheren) Bildung und öffentlichen Kommunikation muss man immer stärkere Tendenzen zur Anglifizierung wahrnehmen. Bleiweis widersetzte sich albernen „Argumenten" für den Vorrang des weit verbreiteten Deutschen, heute ist die vorteilhafte Sprache halt 
Englisch. ${ }^{10}$ Die Sprachwahl und -verwendung waren (und bleiben nach wie vor) gesetzlich nur vage geregelt. Zu Bleiweis' Zeiten konnten z. B. Gerichtsverhandlungen nur von Richtern, Beamten und Verteidigern geführt werden, die das Slowenische gut beherrschten „(nach Thunlichkeit)“, Urteilsbekundungen und ebenso (nach Möglichkeit) Urteilserklärungen sollten auf Slowenisch erfolgen, nicht zuletzt war die Kenntnis des Slowenischen eine der Bedingungen für die Beamtenanstellung: „Zapovedano je, da se mora [...] osebno gledati na to, da prosivci znajo slovenski jezik“. Bleiweis war gegenüber vagen Formulierungen im Erlass des Ministers zurückhaltend: „Ako besedo zmožnost / Thunlichkeit in možnost / Möglichkeit bode tolmačil vsak uradnik sam, je vsa dobra ministrova volja zastonj. [...] Ravnopravnost slovenska ni sicer s tem še ni rešena, pa en korak je spet storjen" (Novice, 26.3.1862, 20/13: 97). Seine Bedenken waren gerechtfertigt, denn die Interpretation der Verordnung wurde den deutschtümlerischen Beamten überlassen, im deren Interesse keinerlei sprachliche Regelungen zugunsten des Slowenischen lagen - unter anderem beklagten sie eine nicht auszuweichende Verdoppelung der Korrespondenz, die für den Staat angeblich zu teuer wäre, ${ }^{11}$ ferner die Notwendigkeit, zusätzliche Beamten anzustellen und die Mangelhaftigkeit des Slowenischen wegen seiner regionalen und dialektalen Vielfältigkeit. Gegenüber derartigen „Erklärungen“ blieb Bleiweis jedoch, wie sonst üblich, nicht zurückhaltend; klar und deutlich verteidigte er die Eigenständigkeit des Slowenischen: „Za Slovence po slovensko! Jezik ni „Modeartikel“ - in se tudi oktrojirati ne da [...], na razumi in omiki ljudstva je vse ležeče“. Langfristig gesehen waren seine Positionen angemessen - die historische gesetzliche Sprachregelung ersetzte er durch eine Nationalidee, wobei die Vereinheitlichung der slowenischen Schriftnorm eine bedeutsame Rolle einnahm. Die nationale Sprache wurde zum äußeren Merkmal der Staatsbildung im Sinne des Vereinigten Sloweniens, laut Bleiweis zum äußeren Merkmal der nationalen Autonomie. Sprachideen der illyrischen Bewegung lehnte er entschieden ab, eine gemeinsame „illyrische“ Literatursprache aller Slawen verstand er lediglich als „,theoretische Annahme“, realisierbar nur bei einer vollständigen physischen und geistigen, emotionalen und gedanklichen Vereinheitlichung aller slawischen Völker (Prijatelj 1937: 47). Angesichts derartig utopischer Ideen sah er die künftige Perspektive des Slowenentums lediglich in der Etablierung einer einheitlichen slowenischen Schriftsprache.

In Bezug auf das Krainische berief er sich lange Zeit auf die normative Grammatik von F. Metelko, bei der späteren Ausgestaltung der einheitlichen slowenischen Sprache betrachtete er allerdings die Grammatik von J. Muršec (MuršEc 1847) als geeignetere Referenz für alle Slowenen. Trotz der Betonung einiger Besonderheiten der steirischen Dialekte war diese dem einheitlichen Slowenisch mehr gerecht als die Grammatiken von P. Dajnko (DAjnko 1824) und F. Metelko (Metelko 1825), welche etliche steirische und krainische Besonderheiten durchzusetzen versuchten.

Bleiweis Wunsch war es, dass die slowenische Sprache alle Slowenen zusammenführen könnte. Die verbindende Funktion der Sprache sah er zunächst jedoch nicht gebunden an die sog. neuen Formen (vgl. JANEžIČ 1854), welche Mitte des 19. Jahrhunderts das Neuslowenische angekündigt hatten und auch in der Übersetzung des Grundgesetzes (Državni zakonik) angewendet wurden.

\footnotetext{
${ }^{10}$ Von der slowenischen Forschungsagentur (ARRS) wird z. B. vorgeschlagen, die gesetzliche Regelung offizieller Verfahren zu ändern, indem das Slowenische in gesamter Dokumentation nicht mehr vertreten wäre (Predlog Zakona 2019: 67). Slowenische Forscher sollten Ihre Programm- und Projektanträge nur noch auf Englisch einreichen, um das jeweilige Prozedere zeitlich und finanziell zu optimieren. Derartiges Verständnis des Slowenischen als Staatssprache ähnelt den Sprachansichten im 19. Jahrhundert, wonach Slowenisch nur für private Kommunikation geeignet wäre.

${ }^{11}$ Wie bekannt klingt diese Meinung! Auch die slowenische Forschungsagentur (ARRS) beruft sich auf die Kostenreduzierung, falls Slowenisch in offiziellen Verfahren nicht mehr vertreten ist (vgl. Anm. 10).
} 
Noch im Jahr 1849 setzte er sich für eine leicht verständliche „reine slowenische Sprache“ ohne die neuen, zu stark illyrischen und fremden Formen (Novice, 12.12.1849, 7/50: 215). Als bedingt geeignet fand er sie nur für die fachsprachlich geprägte Gesetzgebung (Državni zakonik). Neue Formen seien etymologisch fremd und nicht „aus dem Volke genommen“, es ginge um einen geleiteten Eingriff in die Sprachstruktur auf Rechnung der Verständlichkeit. Der Übergang vom Krainischen zum Neuslowenischen war allerdings bereits vor der Einführung neuer Formen anzunehmen; durch die Annäherung der einmaligen Zentral- und Ostslowenischen Schriftsprachen spielte hierbei die Zeitschrift Novice eine bestimmende Rolle (JESENŠEK 2013, JESENŠEK 2018). Allmählich änderte Bleiweis sein Verhältnis zur neuen Sprache, insbesondere nach der Feststellung, dass sie von den meisten Slowenisch Schreibenden problemlos akzeptiert wurden. Dabei richtete er sich nach der praktischen Zweckmäßigkeit: er selbst blieb zunächst bei alten Formen, während bei den Zeitschriftkorrespondenten neue Formen ohne Korrekturen akzeptiert wurden. Auch relativierte er ihr Gewicht für die weitere Entwicklung des Slowenischen; sie seien „Kleinigkeiten“ ohne den Einfluss auf die Vereinheitlichung der Slowenen und Ausgestaltung der einheitlichen slowenischen Schriftsprache (Novice, 30.10.1850, 8/44: 186). Endlich schrieb seit 1853 auch er in der neuen slowenischen Sprache (PRIJATELJ 1937: 91). Eher zögerlich handelte er auch bei der Einführung der Schrift Slovenica, indem er zunächst die Schrift von Metelko (Metelčica) ablehnte und daraufhin die Transkription der Schriften von Bohorič (Bohoričica) und Metelko (Metelčica) in die neue Schrift Slovenica veröffentlichte (Novice, 14.2.1849, 7/7: 30). Dabei berief er sich auf das Urteil der Leser, denen die Sprachnorm anzupassen ist.

\section{SCHLUSS}

Manche Erforscher der literarischen und politischen Geschichte des slowenischen Sprachraumes übertrieben mit der Darstellung eher negativer persönlicher Eigenschaften von Bleiweis. Ein Genius war er bestimmt nicht, seine theoretischen Ansichten waren nicht besonders ausgeprägt, jedoch wurde er mit der Zeit zu einem ausgezeichneten Praktiker. Obgleich er sich für Neuerungen aller Art nur schwer entscheiden konnte, lehnte er sie nie stur oder unargumentiert ab. Mit seiner Zeitschrift Novice beeinflusste er mehr als bestimmend das öffentliche Leben der Slowenen. Unvoreingenommen und in der Einstimmung mit realen Verhältnissen und Umständen äußerte sich dazu der Schriftsteller Janez Trdina:

»Novice« mene od kraja niso dosti veselile, nekaj radi novih črk, katere so se priporočale in uvajale, Se bolj pa radi mnogih besed, ki jih nisem razumel. Ali sčasoma sem se privadil i Husovim črkam i jeziku in sem komaj čakal, da jih dobim v roke; prebral sem verno in pazljivo vsak svoj izvod do Bleiweisove smrti. Mikal me je seveda najbolj leposlovni del, posebno pa pesmi (Trdina 1905: 590-591).

Übersetzt von Vida Jesenšek

\section{DANKSAGUNG}

Der Beitrag entstand im Rahmen des Forschungsprogramms Nr. P6-0156 (Slovensko jezikoslovje, književnost in poučevanje slovenščine, Leitung Prof. Dr. Marko Jesenšek), finanziell unterstützt durch die slowenische Forschungsagentur (Javna agencija za raziskovalno dejavnost Republike Slovenije). 


\section{LITERATUR}

BREZNIK 1933 = BREZnik Anton: O časnikarski slovenščini. Bleiweis. Dom in svet 46/1-2 (1933) 75-82.

BREZNIK 1982 = BREZNIK Anton: Jezikoslovne razprave. Ljubljana, 1982.

DAjnKo 1824 = Dajnko P. Lehrbuch der windischen Sprache. Graz, 1824.

JANEŽIČ 1854 = JANEŽIČ A. Slovenska slovnica za domačo in šolsko rabo. Celovec, 1854.

JeSEnšEK 2013 = JesenšEK Marko: Poglavja iz zgodovine prekmurskega knjižnega jezika. Maribor, 2013.

JeSENŠEK 2018 = JeSENŠEK Marko: Prekmurščina med knjižno normo in narečjem. Maribor, 2018.

Kermauner 1956 = Kermauner Dušan: Pripombe k drugi knjigi: Ivan Prijatelj, Slovenska kulturnopolitična in slovstvena zgodovina 1848-1895. Druga knjiga. Ljubljana, 1956. 499-626.

LEGIŠA 1959 = LEGIŠA Lino: Novice in njih sodelavci. In: LEGIŠA Lino (ured.): Zgodovina slovenskega slovstva. T. 2. Romantika. Ljubljana, 1959. 169-174.

Levec 1880 = Levec Fran: Dr. Janez Bleiweis. In: Slovenske večernice za poduk in kratek čas 35. Celovec, 1880. 8-23.

LevstiK 1858 = Levstik Fran: Napake slovenskega pisanja. Kmetijske in rokodelske novice 16/1-16/28 (1858) 3: [...]-219. [Auch in: Levstik Fran: Levstikovi zbrani spisi IV. Proza II. Ljubljana, 1892. 22-86.]

LOKAR 1909 = LOKAR Janko: Bleiweis in Novičarji v borbi za slovenski jezik in domače slovstvo. In: TOMINŠEK Josip (ured.): Bleiweisov zbornik. (Zbornik Matice Slovenske XI.) Ljubljana, 1909. 1-140.

LoNČAR 1909 = LoNČAR Dragotin: Dr. Janez Bleiweis in njegova doba. In: ToMINŠEK Josip (ured.): Bleiweisov zbornik. (Zbornik Matice Slovenske XI.) Ljubljana, 1909. 141-244.

MaL 1993 = MaL Josip: Zgodovina slovenskega naroda. Celje, 1993.

MARN 1882 = MARn Josip: Novice pa dr. Janez Bleiweis. Jezičnik 20 (1882): 1-74.

Melik 2002 = Melıк Vasilij: Slovenci 1848-1918: razprave in članki. Maribor, 2002.

Metelko 1825 = Metelko F. S. Lehrgebäude der slowenischen Sprache im Königreiche Illyrien und in den benachbarten Provinzen. Laibach, 1825.

Murko 1832 = Murko M. Grammatik der slowenischen Sprache. Graz, 1832.

MuRŠEC 1847 = MuRŠEC J. Kratka slovenska slovnica za pervence. Gradec, 1847.

OrožEN 1996a = OrožEN Martina: Oblikovanje enotnega slovenskega knjižnega jezika. Ljubljana, 1996.

OrožEn 1996b = OrožEn Martina: Poglavja iz zgodovine slovenskega knjižnega jezika. Ljubljana, 1996.

Ротос̌Nıк 1849 = Ротос̌NIK B. Grammatik der slowenischen Sprache. Laibach, 1849.

Predlog Zakona = Predlog zakona o znanstvenoraziskovalni in inovacijski dejavnosti, 2019. https://e-uprava. gov.si/drzava-in-druzba/e-demokracija/predlogi-predpisov/predlog-predpisa.html?id=8644.

Prijatelj 1937 = Prijatelj Ivan: Borba $z a$ individualnost slovenskega jezika v letih 1848-1858. Ljubljana, 1937.

Prijatelj 1955 = Prijatelj Ivan: Slovenska kulturnopolitična in slovstvena zgodovina 1848-1895. Prva knjiga. Ljubljana, 1955.

Prijatelj 1956 = Prijatelj Ivan: Slovenska kulturnopolitična in slovstvena zgodovina 1848-1895. Druga knjiga. Ljubljana, 1956.

$\mathrm{SBL}=$ Slovenski biografski leksikon. Hg. Petra Vide Ogrin. Version 1.0, 2013-12-05. Ljubljana, 2013. https:// www.slovenska-biografija.si/nsbl/.

ŠTrEKELJ 2014 = ŠTREKELJ Karel: Zgodovina slovenskega slovstva III-IV. Ljubljana, 2014.

TominšEK 1904 = TomıNšEK Josip: Bitje in žitje Bleiweisovih »Novic«. Ljubljanski zvon 24/7 (1904) 458: [...]-715.

Tominšek 1909 = TominšEK Josip: Dr. Janez Bleiwei vitez Terstenišk. In: TominšEK Josip (ured.): Bleiweisov zbornik. (Zbornik Matice Slovenske XI.) Ljubljana, 1909. V-LVI.

TOPORIšIČ 1986 = TOPORIšIČ Jože: Portreti, razgledi, presoje. Maribor, 1986. 
Trdina 1905 = Trdina Janez: Moje življenje. Ljubljanski zvon 25 (in Fortsetzungen 1905/1906 unter dem

Titel: Dr. F. Derganc, Janez Trdina, Avtobiografska pisma). http://www.cloverleaf-mall.com/knjige/ moje_zivlenje_trdina.pdf.

VošNJAK 1982 = VošNJAK Josip: Spomini. Ljubljana, 1982.

ŽIGON 1920 = ŽI IGON Avgust: Korytkova pogodba z Blaznikom iz leta 1838 (prispevek k Prešernovi literarni zapuščini). Ljubljanski zvon 40 (1920) 5-12: 462-465.

\author{
MARKO JESENŠEK \\ Faculty of Arts, University of Maribor, Slovenia \\ Slovenian Academy of Sciences and Arts, Ljubljana, Slovenia
}

\title{
The Role of Bleiweis' Newspaper Kmetijske in rokodelske novice in the Formation of a Unified Standard Slovenian Language
}

J. Bleiweis' newspaper Kmetijske in rokodelske novice (Agricultural and Artisanal News), also known simply as Novice (News), played a key role in the creation of a unified Standard Slovenian language by bringing together all Slovenian writers and providing readers with a means to learn about writing and encouraged the reading of Slovenian texts.

The newspaper built on the sense of Slovenian affiliation and the idea of the United Slovenia by reinforcing the unified Standard Slovenian language and unified Slovenian writing called slovenica, rejecting the Illyrian movement and Pan-Slavicism, later somewhat less convincingly with the adoption of new forms that Bleiweis initially established as a defiance against "our pure" and "comprehensible" Slovenian language.

Bleiweis' efforts to establish the use of the Slovenian language in schools and public life made it possible for the Slovenian language to achieve four-part perfection regarding its functional varieties, i.e. expanding from its basic practical and communicative "home environment" to the public sphere, where it functioned easily in journalism, took on the fully-fledged role of a specialist language in the translation of Državni zakonik (the official collection of national rules and regulations) and that of an artistic language also used in Prešeren's poems published in Novice.

As a result of Bleiweis' Novice, schools, newspapers, and books in Slovenia were able to gain public acclaim. Despite the editor maintaining that Novice was an "educational journal for a simple people", it was in fact also a political newspaper that suited intellectuals; it was at the heart of the Slovenian national revival and, as such, opened a public discussion about all the important issues of Slovenism, particularly regarding language, culture, politics, and literature.

Keywords: the history of the Slovenian language, J. Bleiweis, Kmetijske in rokodelske novice, the unification of Standard Slovenian norms, new Slovenian, United Slovenia, formal language, the language of education

Open Access. This is an open-access article distributed under the terms of the Creative Commons Attribution-NonCommercial 4.0 International License (https://creativecommons.org/licenses/by-nc/4.0/), which permits unrestricted use, distribution, and reproduction in any medium for non-commercial purposes, provided the original author and source are credited, a link to the CC License is provided, and changes - if any - are indicated. 